\title{
Assessment of Difference in the Clinical and Microbial Spectrum of Community-Acquired Pneumonia among Egyptian Children Abdelrazek Mohamed Elshiekh ${ }^{1}$, Rabab Mohamed El Behedy ${ }^{1}$, Ghada El Siad Amro'2, Salem Faraj Salem Alayan*1 \\ Departments of ${ }^{1}$ Pediatrics and ${ }^{2}$ Clinical Pathology, Faculty of Medicine, Zagazig University, Egypt \\ *Corresponding author: Salem Faraj Salem Alayan, Email: ayansalem22@gmail.com
}

\begin{abstract}
Background: Community-acquired pneumonia (CAP) is a significant cause of respiratory morbidity and mortality in children, especially in developing countries. Chest radiography and rapid viral testing may be helpful when the diagnosis is unclear.

Objective: This study aimed to find pattern community-acquired pneumonia (CAP) pathogen after introduction of Haemophilus influenzae type B (HIB) vaccine in Egypt and also to provide an update on the evaluation, diagnosis and treatment of CAP in children.

Patients and methods: A cross sectional study that was conducted on 54 patients with pneumonia infection. Participation to this study was offered to all consecutive children aged 2 months to 7 years old presenting with CAP according to the WHO criteria. All cases with CAP were subjected to full history, clinical examination, radiological plan chest X-ray and laboratory and microbiological investigations. Results: The mean age was 1.79 years and mean weight was $9.61 \mathrm{Kg}$, more than half of them were male $(57.4 \%)$. About $94.4 \%$ of the studied group had respiratory distress (RD) Grade I followed 70.4\% had Grade II, 46.3\% had grade III. The mean hemoglobin (Hb) level was 11.06 $\mathrm{gm} / \mathrm{dl}$, mean hematocrit was $32.44 \%$, while mean neutrophil was $6.61 \%$. Additionally, the mean platelets count was $345.81 \times 103 / \mathrm{mm}^{3}$ mean CRP was $43.26 \mathrm{mg} / \mathrm{dl}$, and the median of PCT was 1.13 . All $64.8 \%$ of the studied group had disease less than 1 week ago. Regarding oxygen support $14.8 \%$ needed ventilator. Finally $22.2 \%$ were admitted to ICU. Conclusion: Bacteremia is an unusual complication of CAP in hospitalized children. Our study suggests that approximately $1 \%$ of admitted children with CAP are bacteremic.
\end{abstract}

Keywords: CAP, Egyptian Children, Microbiological Investigations.

\section{INTRODUCTION}

Community-acquired pneumonia (CAP) is a significant cause of respiratory morbidity and mortality in children, especially in developing countries. Worldwide, CAP is the leading cause of death in children younger than five years ${ }^{(\mathbf{1})}$. Factors that increase the incidence and severity of pneumonia in children include prematurity, malnutrition, low socioeconomic status, exposure to tobacco smoke, and child care attendance ${ }^{(2)}$.

The diagnosis of CAP in children is mainly based on clinical symptoms. Tachypnea is the most sensitive sign for predicting pneumonia in children, evident on chest radiography. In children over the age of one month, cough is the commonest symptom. Fever is common but not always present at the time of assessment. Fever alone occurring without cough or respiratory distress may still be due to pneumonia ${ }^{(3)}$.

Determining the etiology of CAP is still difficult in routine clinical settings considering the difficulty in obtaining appropriate lower respiratory tract specimens from children. A considerable seasonal and geographical difference in such etiology has been reported. In most studies, Streptococcus pneumoniae has been the most common etiologic agent identified ${ }^{(4)}$.

Atypical pneumonia is used to describe pneumonia caused by atypical pathogens such as Mycoplasma pneumoniae, Chlamydia pneumoniae, Legionella pneumophila, and others. The clinical course among children infected with these pathogens is different from that of other bacterial or viral infection, most of them progress slowly and have no specific symptoms. Many studies reported that Mycoplasma pneumoniae is the most common atypical pathogen found among children with CAP, followed by Chlamydia pneumoniae ${ }^{(5,6)}$.

While recognizing the etiology of communityacquired pneumonia is necessary for formulating local antimicrobial guidelines, limited data are published about this etiology in Egyptian pediatric patients ${ }^{(7)}$.

This study aimed to find pattern communityacquired pneumonia (CAP) pathogen after introduction of HIB vaccine in Egypt. Also, it aimed to provide an update on the evaluation, diagnosis and treatment of CAP in children.

\section{PATIENTS AND METHODS}

A cross sectional study was conducted on 54 patients with pneumonia infection, at Zagazig University Hospital. Participation to this study was offered to all consecutive children aged 2 months to 10 years old presenting with CAP according to the WHO criteria.

Inclusion and exclusion criteria: Children with CAP were 31 males and 23 females, their age ranged from 2 
month to 7 years with mean age of 1.79 years. While, children aged less than two months and more than 10 years and children not received HIB vaccine were excluded.

\section{Technique:}

All children were subjected to full history taking, local and systemic clinical examination and radiological examination.

Laboratory investigation: Diagnostic tests including complete blood count (CBC), C-reactive protein (CRP), serum procalcitonin (PCT), blood culture and urine analysis.

Samples: Sputum samples were obtained by aspirating the nasopharynx through the nostrils with a disposable mucus extractor or by expectoration if the child was old enough to produce an adequate sputum sample. Cough swab was done.

\section{Processing of specimens:}

Respiratory samples were subjected to inoculation on blood agar, heated blood agar and MacConkey's agar media. Direct smear staining with Gram stain for microscopic examination was performed.

All sputum cultures were screened for interpretability; only those with $>25$ leukocytes $<10$ epithelial cells per low power field were selected. Inoculated blood and MacConkey's agar plates were incubated at $37^{\circ} \mathrm{C}$ aerobically while inoculated heated blood agar plates were incubated at $\mathrm{CO}_{2} 10 \%$ by the candle jar method. Blood cultures were incubated overnight at $37^{\circ} \mathrm{C}$. A blind subculture was done on blood agar plates after overnight incubation; if no growth was obtained from respiratory and blood cultures were completely identified using standard techniques.

\section{Gastric aspirates culture:}

In a child with suspected pulmonary tuberculosis (TB), the cough may be scarce or nonproductive. Therefore, the best test for diagnosis is an early-morning gastric aspirate (GA) sent for acid-fast bacilli (AFB) stain, culture and if available. GA should be obtained by first placing a nasogastric (NG) tube the night before sample collection; a sample is aspirated the first thing the following morning, before ambulation and feeding. This should be repeated on 3 consecutive mornings.

\section{Chest radiography:}

Chest radiographs (Outpatient): Posteroanterior chest radiographs were not required to confirm the CAP diagnosis but they were performed for patients with suspected or recorded hypoxia or those with significant respiratory distress, as well as for patients who failed to respond to initial antimicrobial treatment to check for pneumonia complications.

Chest radiographs (Inpatient): chest radiographs were performed for all patients hospitalized with CAP to outline the size and characteristics of parenchymal infiltrates, and to recognize complications of pneumonia that may require prompt intervention rather than antimicrobial therapy.

\section{Ethical consent:}

An approval of the study was obtained from Zagazig. University Academic and Ethical Committee. Every parent of each patient signed an informed written consent for acceptance of participation in the study. This work has been carried out in accordance with The Code of Ethics of the World Medical Association (Declaration of Helsinki) for studies involving humans.

\section{Statistical analysis}

All data were analyzed using Minitab 17.0 statistical software (Minitab Inc., Pennsylvania U.S.A). Continuous variables were expressed as the mean $\pm \mathrm{SD}$, range, median, and interquartile range (IQR) and the categorical variables were expressed as a number (percentage). $\mathrm{Chi}^{2}$ test was used to compare categorical variables. All tests were two sided. $\mathrm{P}<0.05$ was considered statistically significant and $\mathrm{p}<0.01$ was considered highly statistically significant.

\section{RESULTS}

The demographic characteristics of the studied patients are shown in table 1.

Table (1): Demographic characteristics of the studied group

\begin{tabular}{|c|c|c|}
\hline Variable & \multicolumn{2}{|c|}{$(\mathrm{n}=54)$} \\
\hline $\begin{aligned} \text { Age: } & \text { (years) } \\
& \text { Mean } \pm \text { SD } \\
& \text { Range } \\
& \text { Median }(\mathrm{IQR})\end{aligned}$ & \multicolumn{2}{|c|}{$\begin{array}{c}1.79 \pm 1.75 \\
2 \text { months }-7 \text { years } \\
1.42(0.42-2.67)\end{array}$} \\
\hline Weight: (Kg)- Mean \pm SD & \multicolumn{2}{|c|}{$9.61 \pm 4.86$} \\
\hline Sex: $(\%)$ & No & $\%$ \\
\hline Male & 31 & 57.4 \\
\hline Female & 23 & 42.6 \\
\hline
\end{tabular}

SD: Standard deviation, IQR: Interquartile range.

Regarding symptoms most of the studied group had RD grade I and II. Most frequent symptoms among the studied group were diminishing in air entry (Table 2). 
Table (2): Symptoms among the studied group

\begin{tabular}{|c|c|c|c|}
\hline \multirow{2}{*}{\multicolumn{2}{|c|}{ Variable }} & \multicolumn{2}{|c|}{$\overline{(2 n=54)}$} \\
\hline & & No & $\%$ \\
\hline \multirow{4}{*}{$R D:$} & Grade I & 51 & 94.4 \\
\hline & Grade II & 38 & 70.4 \\
\hline & Grade III & 25 & 46.3 \\
\hline & Grade IV & 14 & 25.9 \\
\hline \multirow{2}{*}{ Cough: } & Yes & 35 & 64.8 \\
\hline & No & 19 & 35.2 \\
\hline \multirow{2}{*}{ Wheeze: } & Yes & 19 & 35.2 \\
\hline & No & 35 & 64.8 \\
\hline \multirow{2}{*}{ Refuse of feeding: } & Yes & 31 & 57.4 \\
\hline & No & 23 & 42.6 \\
\hline \multirow{2}{*}{ Cyanosis: } & Yes & 18 & 33.3 \\
\hline & No & 36 & 66.7 \\
\hline \multirow{2}{*}{ Rapid Breaths: } & Yes & 40 & 74.1 \\
\hline & No & 14 & 25.9 \\
\hline \multirow{2}{*}{ Fine crepitation: } & Yes & 39 & 72.2 \\
\hline & No & 15 & 27.8 \\
\hline \multirow{2}{*}{$\begin{array}{l}\text { Diminished air } \\
\text { entry: }\end{array}$} & Yes & 51 & 94.4 \\
\hline & No & 3 & 5.6 \\
\hline
\end{tabular}

RD: Respiratory distress [4 grades] (Grade I: tachypnea + nasal flaring; Grade II: grade I + chest retraction [retraction = intercostal, sub-costal, supra-sternal] Grade III: grade II + grunting; and Group IV = grade III + cyanosis).

The laboratory findings of the studied patients are shown in table 3.

Table (3): Laboratory findings among the studied group

\begin{tabular}{|c|c|}
\hline Variable & $(\mathbf{n}=54)$ Mean \pm SD \\
\hline$H b:(\mathrm{gm} / \mathrm{dl})$ & $11.06 \pm 1.16$ \\
\hline Hematocrit: (\%) & $32.44 \pm 4.21$ \\
\hline WBCs: $\left(x 10^{3} / \mathrm{mm}^{3}\right)$ & $13.83 \pm 3.86$ \\
\hline Lymphocyte: (\%) & $3.75 \pm 0.7$ \\
\hline Neutrophil: (\%) & $6.61 \pm 1.64$ \\
\hline Platelets: $\left(x 10^{3} / \mathrm{mm}^{3}\right)$ & $345.81 \pm 33.14$ \\
\hline CRP: $(\mathrm{mg} / \mathrm{L})$ & $43.26 \pm 3.43$ \\
\hline $\begin{array}{c}\text { Urine analysis: (No, and \%) } \\
\text { Normal } \\
\text { Abnormal }\end{array}$ & $\begin{array}{c}49(90.7 \%) \\
5(9.3 \%)\end{array}$ \\
\hline \begin{tabular}{|l} 
Pus cells: \\
$\quad<5$ \\
$\quad 5-10$ \\
$>10-15$ \\
$>15$
\end{tabular} & $\begin{array}{c}49(90.7 \%) \\
3(5.6 \%) \\
1(1.9 \%) \\
1(1.9 \%) \\
\end{array}$ \\
\hline $\begin{aligned} \text { RBCs: } & \\
& <5 \\
& 5-10 \\
& >10\end{aligned}$ & $\begin{array}{l}51(94.4 \%) \\
2(3.7 \%) \\
1(1.9 \%)\end{array}$ \\
\hline $\begin{array}{c}\text { Nitrate: } \\
+\mathrm{ve} \\
-\mathrm{ve}\end{array}$ & $\begin{array}{c}50(92.6 \%) \\
4(7.4 \%)\end{array}$ \\
\hline $\begin{array}{c}\text { Bacteria: } \\
- \\
+ \\
++ \\
+++ \\
\end{array}$ & $\begin{array}{c}49(90.7 \%) \\
1(1.9 \%) \\
2(3.7 \%) \\
1(3.7 \% \\
\end{array}$ \\
\hline
\end{tabular}

$\mathrm{Hb}$ : hemoglobin WBCs: white blood cells, CRP: C-reactive protein RBCs: Red blood cells 
Most of the studied group had disease less than 1 week ago. Regarding oxygen support $14.8 \%$ needed ventilator. Finally $22.2 \%$ were admitted to ICU (Table 4).

Table (4): Duration, oxygen support, ICU admission among the studied group

\begin{tabular}{||c|c|c|}
\hline \multicolumn{1}{|c|}{ Variable } & \multicolumn{2}{|c|}{ (n= 54) } \\
\hline \hline & No & \% \\
\hline Duration: & 35 & 64.8 \\
< 1 week & 15 & 27.8 \\
1 - weeks & 4 & 7.4 \\
> 2 weeks & 46 & 85.2 \\
\hline Oxygen support: & 8 & 14.8 \\
Nasal O & & 22.2 \\
Ventilator & 12 & 77.8 \\
\hline ICU: & 42 & Yes \\
No & & . \\
\hline
\end{tabular}

Among 8 cases who had sputum culture, 5 cases (62.5\%) had +ve culture (Staph). Also among 46 cases had gastric aspiration $80.4 \%$ had +ve results. Most frequent organism founded were klebsilla and E. coli. As regard blood culture, $66.7 \%$ was - ve, $11.1 \%$ was contaminated and $22.2 \%$ was +ve (Table 5).

Table (5): Serology findings among the studied group

\begin{tabular}{|c|c|c|}
\hline Variable & No & \% \\
\hline \hline Sputum culture: $(\boldsymbol{n}=\mathbf{8})$ & & \\
Positive $($ Staph $)$ & 5 & 62.5 \\
Negative & 3 & 37.5 \\
\hline Gastric aspiration: $(\boldsymbol{n}=\mathbf{4 6})$ & $\mathbf{3 7}$ & $\mathbf{8 0 . 4}$ \\
Positive & 14 & 30.4 \\
Klebsilla & 9 & 19.6 \\
E. coli & 5 & 10.9 \\
Staph & 3 & 6.5 \\
Pseudomonas & 6 & 13 \\
Candida & $\mathbf{9}$ & $\mathbf{1 6 . 9}$ \\
Negative & & \\
Blood culture: $(\boldsymbol{n}=\mathbf{5 4})$ & $\mathbf{1 3}$ & $\mathbf{2 4 . 1}$ \\
Positive & 8 & 14.8 \\
Klebsilla & 3 & 5.6 \\
E. coli & 2 & 3.7 \\
Staph & $\mathbf{3 6}$ & $\mathbf{6 6 . 7}$ \\
Negative & $\mathbf{5}$ & $\mathbf{9 . 3}$ \\
\hline
\end{tabular}

There was no statistical significance relation between blood culture and sputum and gastric culture among the studied group (Table 6).

Table (6): Relation between blood culture and sputum and gastric culture among the studied group

\begin{tabular}{|c|c|c|c|c|c|c|c|c|}
\hline \multirow{2}{*}{ Variable } & \multirow{2}{*}{$\mathbf{N}$} & \multicolumn{2}{|c|}{ - ve $(n=36)$} & \multicolumn{2}{|c|}{ Contaminated $(\mathrm{n}=5)$} & \multicolumn{2}{|c|}{+ ve $(n=13)$} & \multirow{2}{*}{$\begin{array}{c}\mathbf{P} \\
\text { value }\end{array}$} \\
\hline & & No & $\%$ & No & $\%$ & No & $\%$ & \\
\hline $\begin{array}{c}\text { Sputum culture: } \\
+\mathrm{ve} \\
-\mathrm{ve}\end{array}$ & $\begin{array}{l}5 \\
3 \\
\end{array}$ & $\begin{array}{l}4 \\
2\end{array}$ & $\begin{array}{l}80.0 \\
66.7\end{array}$ & $\begin{array}{l}0 \\
0\end{array}$ & $\begin{array}{l}0.0 \\
0.0\end{array}$ & $\begin{array}{l}1 \\
1\end{array}$ & $\begin{array}{l}20.0 \\
33.3\end{array}$ & 0.067 \\
\hline $\begin{aligned} \text { Gastric: } & \\
& +\mathrm{ve} \\
& -\mathrm{ve}\end{aligned}$ & $\begin{array}{c}37 \\
9\end{array}$ & $\begin{array}{c}27 \\
3\end{array}$ & $\begin{array}{l}73.0 \\
33.3\end{array}$ & $\begin{array}{l}1 \\
4\end{array}$ & $\begin{array}{c}2.7 \\
44.4\end{array}$ & $\begin{array}{l}9 \\
2\end{array}$ & $\begin{array}{l}24.3 \\
22.2\end{array}$ & 0.13 \\
\hline
\end{tabular}




\section{DISCUSSION}

In the current study, a total of 54 children $(57.4 \%$ boys) were included. Patients' mean age was 1.79 years and it ranged from 2 month to 7 years, and weight ranged from 3 to $21 \mathrm{Kg}$ with a mean of $9.61 \mathrm{Kg}$. These findings were in agreement with the study of Hatem $\boldsymbol{e t}$ al. ${ }^{(8)}$, and Tannous et al. ${ }^{(9)}$ who showed that $60.4 \%$ of patients were males and $39.5 \%$ were females. On the contrary, Mathew et al. ${ }^{(10)}$ found that there were no major differences in gender.

As regarding symptoms among children with CAP, the current study shows that $94.4 \%$ of the studied group had respiratory distress (RD) grades classified into 4 grades. $94.4 \%$ had Grade I, $70.4 \%$ had Grade II, 46.3\% had Grade III, and 25.9\% Grade IV. However, most frequent symptoms among the studied group were diminishing in air entry followed by rapid breaths and fine crepitation $(94.4 \%, 74.1 \%$ and $72.2 \%$ respectively). These findings were going with the study of Sweet $\boldsymbol{e t} \boldsymbol{a l}$. (11). The study of Hatem et al. ${ }^{(8)}$ elucidated the most common sign, which was diminished air entry in $97.9 \%$ followed by tachypnea in $85.4 \%$ then oxygen support in $72.9 \%$, grunting in $60.4 \%$, fine crepitations in $56.2 \%$, wheezes in $54.1 \%$, cyanosis in $37.5 \%$ with need of ventilator support in cases of bacterial pneumonia in $20.8 \%$.

Respiratory distress describes a symptom complex representing a heterogeneous group of illnesses. As such, respiratory distress is often defined as a clinical picture based on observed signs and symptoms irrespective of etiology ${ }^{(\mathbf{1 2})}$. Clinical symptoms most commonly cited as indicators of respiratory distress include tachypnea ${ }^{(\mathbf{1 3})}$, nasal flaring ${ }^{(14)}$, grunting, retractions (subcostal, intercostal, supracostal, jugular), and cyanosis ${ }^{(\mathbf{1 5})}$. Other symptoms include apnea, bradypnea, irregular (seesaw) breathing, inspiratory stridor, wheeze and hypoxia ${ }^{(16)}$.

A study of children presenting to emergency departments in Brazil with cough and tachypnea in which $14.3 \%$ of films $(n=182)$ were positive reported a kappa of 0.70 (95\% CI 0.56-0.83) for two radiologists (17). It appears this study did not use a panel for discordant films.

As regard laboratory findings in all CAP patients, the current study revealed that, the mean $\mathrm{Hb}$ level were $11.06 \mathrm{gm} / \mathrm{dl}$; while mean hematocrit was $32.44 \%$. Also mean WBCs count was $13.83 \times 10^{3} / \mathrm{mm}^{3}$ and mean lymphocyte was $3.75 \%$; while mean neutrophil count was $6.61 \%$. Additionally, the mean platelets count was $345.81 \times 10^{3} / \mathrm{mm}^{3}$, the study showed increased CRP and PCT, with mean CRP was $43.26 \mathrm{mg} / \mathrm{L}$ and the median of PCT was 1.13 (IQR 0.98-1.29) ng/ml. Additionally, there was $9.3 \%$ of CAP children showed abnormal urine analysis. Vuong et al. ${ }^{(18)}$ reported that white blood cell (WBC) count $<15,000 /$ micro L suggests a nonbacterial etiology, except in the severely ill patient, who also may be neutropenic and have a predominance of immature cells. WBC $>15,000 /$ micro $\mathrm{L}$ is suggestive of pyogenic bacterial disease. However, children with $\mathrm{M}$. pneumoniae, influenza, or adenovirus pneumonia also may have WBC $>15,000 /$ micro L. Peripheral eosinophilia may be present in infants with a febrile pneumonia of infancy, typically caused by Chlamydia trachomatis. On the contrary, Nademi et al. (19) concluded that, the degree of temperature and WBC count were poor predictors of the serious illness and they can't replace clinical assessment in child with fever. Virkki et al. ${ }^{(20)}$ stated that patients with increase WBCs don't differentiate between viral and bacterial pneumonia.

In the study of Jiao et al. ${ }^{(21)}$, in compliance with clinical manifestations of CAP, they were able to differentiate bacterial infected CAP patients based on the measured PCT, CRP and WBC levels; with this predetermined, positive cut-off range of PCT $>0.05 \mathrm{ng} / \mathrm{ml}$, $\mathrm{CRP}>8 \mathrm{mg} / \mathrm{L}$ and $\mathrm{WBC}>10 \times 101^{2} / \mathrm{L}$ in CAP patients. They found the bacteria (Streptococcus pneumonia and Staphylococcus aureus) infected patients of both age groups ( $<5 \mathrm{y}$ and $>5 \mathrm{y})$ showed increased PCT $(0.135$ $0.142 \mathrm{ng} / \mathrm{ml})$, CRP $(8.268-9.216 \mathrm{mg} / \mathrm{L})$ and $\mathrm{WBC}$ $\left(10.852-11.752 * 10^{12} / \mathrm{L}\right)$ levels compared with the virus (majorly respiratory syncytial virus and adenovirus) and MP infected groups. Also, the Mycoplasma-infected patients who showed increased PCT (0.095$0.104 \mathrm{ng} / \mathrm{ml}$ ) levels compared with the virus infected groups did not show any significant changes in either CRP or WBC levels. This indeed could facilitate an early diagnosis and treatment of bacteria and/or Mycoplasma mediated CAP over virus mediated CAP. Virkki et al. ${ }^{(20)}$ found that difference in CRP level of $>40 \mathrm{mg} / \mathrm{L}, 80 \mathrm{mg} / \mathrm{L}, 120 \mathrm{mg} / \mathrm{L}$ were significant although the sensitivity for detecting bacterial pneumonia was too low for use in clinical practice.

Some reports have shown that serial PCT measurement was useful for predicting the prognosis of CAP patients ${ }^{(22,23)}$. One study showed that nonsurvivors had a small relative increase in PCT values from admission to day 3, whereas survivors had a decrease in PCT values from admission to day $3{ }^{(24)}$. Lee et al. ${ }^{(25)}$ found another reason for the different results is that changes in PCT and CRP levels from admission to day 3 or day 7 were used, not from respective measurement points on admission, day 3 , and day 7 . In the treatment of hospitalized CAP patients Ito et al. ${ }^{(26)}$ usually assess initial treatment efficacy within 72 hour using some clinical biomarkers, symptoms, findings on chest X-ray, and inflammatory biomarkers.

Ito et al. ${ }^{(26)}$ reported WBC count, neutrophil count, CRP, and PCT are generally not useful to discriminate non-severe from severe disease in children with CAP, although CRP and PCT may have some utility in predicting the most severe outcomes.

The use of blood inflammatory biomarkers to distinguish bacterial from viral CAP has been investigated. PCT and CRP have shown some value in the identification of bacterial infections ${ }^{(27)}$, but it has 
not been established yet a relevant clinical cut-off point for their use ${ }^{(28)}$.

As regard duration of disease, oxygen support, and ICU admission among all CAP children, the current study revealed that, $64.8 \%$ of the studied group had disease less than 1 week. Regarding oxygen support $14.8 \%$ needed ventilator. Additionally, 22.2\% were admitted to ICU. These findings were in agreement with Nascimento-Carvalho ${ }^{(29)}$ and Shia et al. ${ }^{(30)}$.

As regard serological findings, the current study revealed that, among 8 cases who had sputum culture 5 cases $(62.5 \%)$ had +ve culture. Staph was the most common pathogen detected in sputum. Also among 46 cases who had gastric aspiration $80.4 \%$ had +ve results and the most frequent organisms found were Klebsiella and E. coli (30.4\% and $19.6 \%)$ respectively. As regard blood culture, $66.7 \%$ were -ve, $11.1 \%$ were contaminated and $22.2 \%$ were +ve (most frequent organism was Staph 13\%). The study of Hatem et al. ${ }^{(9)}$ revealed that most of the identified microbes were isolated from culture of respiratory specimens and blood. They found that $S$. aureus $35.4 \%$ was the most common pathogen detected in sputum regardless the age group, candida was $12.5 \%$, Klebsiella was $8.33 \%$, and Pseudomonus was $4.16 \%$. Also, the blood culture gave negative results in most cases of pneumonia $62.5 \%$. S. aureus was the most common pathogen detected in blood culture in positive cases regardless the age group $18.7 \%$, E. coli was $6.25 \%$, and Klebsiella was $2.08 \%$.

The results of Hatem et al ${ }^{\left({ }^{(9)}\right.}$ demonstrated blood culture yield was limited $27.08 \%$. Contamination was found in $10.4 \%$. Most investigators suggest that blood cultures are likely to be unhelpful for management and can be omitted especially in mild and moderate CAP for cost-benefit reasons, while others still find that positive blood cultures can guide antibiotic therapy to narrow spectrum.

The study of Youssef $\boldsymbol{e t}$ al. ${ }^{(31)}$ included a total of 380 blood cultures were obtained, including 23 patients who had repeated blood cultures. Fifteen (3.9\%) cultures were positive for bacterial growth; five $(1.3 \%)$ were considered true positives while the other $10(2.6 \%)$ were treated as contaminants. They concluded that, the prevalence of bacteremia among children admitted for CAP is low. The use of routine blood cultures should be reserved for children with moderate to severe pneumonia.

As regard the relation between blood culture and sputum and gastric culture among all children CAP patients, the current study revealed that there was no statistical significance relation between blood culture and sputum and gastric culture among the studied group. These findings were in accordance with the study of Youssef et al. ${ }^{(31)}$.

Although, the prevalence of bacteremia among children admitted for CAP is low. The use of routine blood cultures should be reserved for children with moderate to severe pneumonia. Further studies are required to better risk-stratify children with $\mathrm{CAP}^{(\mathbf{3 1})}$.

\section{CONCLUSION}

S. aureus in sputum culture, Klebsiella, E. coli, Staph, Pseudomonas, and Candida in gastric aspiration and Klebsiella, E. coli, and Staph in blood culture were the most detected causative agents identified in the study

Financial support and sponsorship: Nil.

Conflict of interest: Nil.

\section{REFERENCES}

1. Black R, Cousens S, Johnson H (2010): Child Health Epidemiology Reference Group of WHO and UNICEF, et al. Global, regional, and national causes of child mortality in 2008: a systematic analysis. Lancet, 375(9730):1969-1987.

2. Stuckey-schrock K, Hayes B, George C (2012): Community-acquired pneumonia in children. Am Fam Physician, 86(7):661-667

3. Yadav R, Kumar D, Singh A et al. (2020): Clinical and microbial spectrum of community-acquired pneumonia in children of north India. Tropical Doctor, 20:1-6

4. Jones B, Tay E, Elikashvili I et al. (2016): Feasibility and safety of substituting lung ultrasonography for chest radiography when diagnosing pneumonia in children: A randomized controlled trial. Chest, 12:131-138.

5. Chen Z, Ji W, Wang Y (2013): Epidemiology and associations with climatic conditions of Mycoplasma pneumoniae and Chlamydophila pneumoniae infections among Chinese children hospitalized with acute respiratory infections. Ital J Pediatr., 39:34-39.

6. Le Huong $P$, Hien $P$, Lan $\mathbf{N}$ et al. (2014): First report on prevalence and risk factors of severe atypical pneumonia in Vietnamese children aged 1-15 years. BMC Public Health, 14:1304-8.

7. El Seify M, Fouda E, Ibrahim H et al. (2016): Microbial etiology of community-acquired pneumonia among infants and children admitted to the Pediatric Hospital, Ain Shams University. Eur J Microbiol Immunol., 6 (3): 206-214.

8. Hatem M, Seham F, Asmaa M et al. (2019): Microbial etiology of community acquired pneumonia among infants and children admitted to Zagazig University Pediatric Hospital. ZUMJ., 25(6): 809-816

9. Tannous R, Haddad R, Torbey P (2020): Management of community-acquired pneumonia in pediatrics: Adherence to Clinical Guidelines. Front Pediatr., 8:302-306.

10. Mathew J, Singhi S, Ray P et al. (2015): Etiology of community acquired pneumonia among children in India: prospective, cohort study. Journal of Global Health, 5(2): 418-422.

11. Sweet L, Keech C, Klein N et al. (2017): Respiratory distress in the neonate: Case definition \& guidelines for data collection, analysis, and presentation of maternal immunization safety data. Vaccine, 35(48): 6506-6517.

12. Qian L, Liu C, Guo Y et al. (2010): Current status of neonatal acute respiratory disorders: a one-year prospective survey from a Chinese neonatal network. Chin Med J., 123(20):2769-2775. 
13. Hermansen C, Mahajan A (2015): Newborn respiratory distress. Am Fam Physician, 92(11):9941002.

14. Reuter S, Moser C, Baack M (2014): Respiratory distress in the newborn. Pediatr Rev., 35(10):417-428.

15. Swarnkar K, Swarnkar M (2015): Neonatal respiratory distress in early neonatal period and its outcome. Int J Biomed Adv Res., 6(9):643-647.

16. Pramanik A, Rangaswamy N, Gates T (2015): Neonatal respiratory distress: a practical approach to its diagnosis and management. Pediat Clin N Am., 62:453469.

17. Castro A, Nascimento-Carvalho C, Ney-Oliveira F et al. (2006): Pulmonary infiltrate among children with cough and tachypnea. Pediatr Infect Dis J., 25(8):75762.

18. Vuong J, Qiu Y, La M et al. (2014): Reference intervals of complete blood count constituents are highly correlated to waist circumference: should obese patients have their own "normal values?" Am J Hematol., 89:671-76.

19. Nademi Z, Clark J, Richards C et al. (2001): The causes of fever in children attending hospital in the north England". J Infect., 43(4):221-5.

20. Virkki R, Juven T, Rikalainen H et al. (2002): Differentiation of bacterial and viral pneumonia in children. Thorax, 57: 438-441.

21. Jiao F, Chen J, Wang M et al. (2019): Determination of procalcitonin, C-reactive protein and white blood cell levels to diagnose community-acquired pneumonia (CAP) The Indian Journal of Pediatrics, 86:763-67.

22. Tamura M, Watanabe M, Nakajima A et al. (2014): Serial quantification of procalcitonin (PCT) predicts clinical outcome and prognosis in patients with community-acquired pneumonia (CAP). J Infect Chemother., 20: 97-103

23. Zhydkov A, Christ-Crain M, Thomann $\mathbf{R}$ et al. (2015): ProHOSP Study Group. Utility of procalcitonin, C-reactive protein and white blood cells alone in combination for the prediction of clinical outcomes in community-acquired pneumonia. Clin Chem Lab Med., 53: 559-566.

24. Schuetz P, Suter-Widmer I, Chaudri A et al. (2011) Procalcitonin-guided antibiotic therapy and hospitalisation in patients with lower respiratory tract infections (ProHOSP) Study Group. Prognostic value of procalcitonin in community-acquired pneumonia. Eur Respir J., 384-392

25. Lee J, Hwang S, Shim J et al. (2010): Clinical significance of serum procalcitonin in patients with community-acquired lobar pneumonia. Korean J Lab Med., 30:406-13.

26. Ito A, Ito I, Inoue D et al. (2020): The utility of serial procalcitonin measurements in addition to pneumonia severity scores in hospitalised community-acquired pneumonia: A multicentre, prospective study. International Journal of Infection Disease, 92:228-233

27. Berg A, Inchley C, Fjaerli H et al. (2017): Clinical features and inflammatory markers in pediatric pneumonia: a prospective study. Eur J Pediatr., 176:629-638.

28. Katz S, Williams D (2018): Pediatric communityacquired pneumonia in the United States: changing epidemiology, diagnostic and therapeutic challenges, and areas for future research. Infect Dis Clin North Am., 32:47-63.

29. Nascimento-Carvalho C (2020): Community-acquired pneumonia among children: the latest evidence for an updated management. J Pediatr (Rio J), 96 (1):29-38.

30. Shia T, Chena C, Huang $L$ et al. (2020): Risk factors for mortality from severe community-acquired pneumonia in hospitalized children transferred to the pediatric intensive care unit. Pediatric and Neonatology, 61 (6):577-583

31. Youssef A, Fanous M, Siddiqui F et al. (2020): Value of blood cultures in the management of children hospitalized with community-acquired pneumonia. Cureus, 12(5): 8222-26. 